\title{
Noise controlled semiconductor optical amplifier based on lateral cavity laser
}

\author{
K. Carney, R. Lennox, R. Maldonado-Basilio, S. Philippe, L. Bradley and P. Landais
}

Experimental characterisation of a novel noise-controlled semiconductor optical amplifier (NCSOA) is presented. The design utilises grooves etched parallel to the active waveguide, at the output sections of the NCSOA, so as to induce lasing laterally to the propagation axis. This clamps the carrier density in a relevant region, allowing for the engineering of a specific longitudinal carrier density profile, corresponding to an improved noise figure performance. Results have demonstrated the effectiveness of carrier density profile engineering as a means of reducing the noise figure in semiconductor optical amplifiers.

Introduction: Semiconductor optical amplifiers (SOAs) are currently of great interest to researchers in the area of optical communications. They have the potential to form the backbone of future alloptical networks and may be utilised for switching and processing applications owing to their nonlinear behaviour when saturated [1]. However, a major draw-back hindering their application as linear amplifiers is an inherently high noise figure (NF), generally in the region of $7-11 \mathrm{~dB}$ for most commer-cially available SOAs [2], when compared with established technologies such as erbiumdoped fibre amplifiers. A number of different approaches have been adopted to reduce this value, including the use of holding beams [3] and manipulation of the confinement factor. Values as low as $4.5 \mathrm{~dB}$ have been reported [4].

A noise-controlled semiconductor optical amplifier (NCSOA) has been proposed and fabricated for testing. This first generation com-ponent uses gain clamping, produced via lateral lasing, to engineer the longitudinal carrier density profile of the active region of the SOA. The profile is chosen to optimise the NF characteristics of the SOA. Theory: The overall NF of a chain of linear amplifiers is driven by the NF of the amplifiers at the front of the chain [5]. This is demonstrated by the Friis equation:

$$
N F_{\text {total }}=N F_{1}+\frac{N F_{2}-1}{G_{1}} \ldots \ldots+\frac{N F_{n}-1}{G_{n-1}}
$$

where NFn and Gn are the noise figure and gain in amplifier n, respectively.

This idea can be applied to a single SOA by considering the SOA as an analogous sectioned system [6]. To reduce the overall NF of the SOA, it is necessary to maintain a lower NF at the input sections than at the output. In general, the NF is proportional to the population inversion factor, $\mathrm{n}_{\mathrm{sp}}$, and inversely proportional to gain. The closer the value of $n_{\text {sp }}$ to 1 in a given section the lower NF in that section, where the population inversion factor can be calculated using the following equation:

$$
n_{s p}=\frac{N_{2}}{N_{2}-N_{1}}
$$

where $\mathrm{N} 2$ and $\mathrm{N} 1$ represent the fractional carrier density in the excited energy level and in the ground state, respectively. Increasing the carrier density in a section of the SOA leads to a larger population inversion, i.e. a larger value for $\mathrm{N} 2$. This has the effect of reducing $\mathrm{n}_{\mathrm{sp}}$ in that section, and consequently reducing its NF. Thus it is clearly desirable to have high carrier density in the input sections of the SOA, and lower carrier density at the output. Conversely, increasing the carrier density at the output and decreasing it at the input should increase the NF. Engineering this carrier density profile is achieved by etching a slot pattern in the cladding along a fraction of the active region of the SOA. The slots form a lateral cavity. At a certain bias current lasing occurs in the cavity, clamping the 
carrier density within this region. The slots are positioned along the output sections of the SOA in order to achieve the desired carrier density profile. Simulations have shown that embedding this cavity along $40-60 \%$ of the length of the SOA length at the output end of the device yields the lowest NF [7], with the consequence of a reduced gain. The low carrier density in this section prevents amplification of spontaneous emission reflected at the output facet, travelling towards the non-gain clamped section of the device, keeping the carrier density in the front region high. In other words, for a given bias current, this SOA achieves a higher carrier density at the input than at the output. This method is distinguished from other gain clamping schemes, such as linear SOAs, by virtue of the fact that the lasing cavities in this concept do not extend along the full length of the device, clamping only a portion of the waveguide and allowing the carrier density to remain high at the front of the device $[8,9]$.

Device under test: The noise controlled device under test is a multi-quantum well InGaAsP/InP SOA (giving a highly TE polarised output), $1 \mathrm{~mm}$ in length with an $800 \mu \mathrm{m}$ active region. The lateral cavity extends over $450 \mu \mathrm{m}$ at the output end of the waveguide. The cavity is formed by two Bragg mirrors, consisting of InP layers, with an $80 \mathrm{~nm}$ airgap between each layer, the thickness of which is chosen to give a maximum reflection at $\lambda=1480 \mathrm{~nm}$. The SOA is temperature controlled at 208C. Amplified spontaneous emission (ASE) spectra from both the input (without lateral cavity) and output (with lateral cavity) facets are collected using a lens and stage combination and coupled to an optical spectrum analyser (OSA) with a resolution bandwidth of $0.06 \mathrm{~nm}$. The ASE peak $(1490 \mathrm{~nm})$ is at the Bragg wavelength of the mirror to facilitate achieving lasing conditions. Fig. 1 shows the collected optical power measured from both facets against supplied bias current. A difference in power from the opposite facets of the SOA can clearly be seen. This is a consequence of the lateral cavity at the output facet. The feature seen in the data for the input facet at high bias current is caused by temperature instability. The optical gain of the SOA, against both signal wavelength and output power at a bias current of $250 \mathrm{~mA}$, is shown in Fig. 2. From these measurements it can be observed that the maximum gain is $8.5 \mathrm{~dB}$, while the saturation output power is $4 \mathrm{dBm}$ (the point at which the gain is reduced by $3 \mathrm{~dB}$ owing to the depletion of charge carriers by the input signal) with a gain bandwidth of $70 \mathrm{~nm}$.

Experiment: Characterisation of the noise figure is carried out using a free space setup. The input signal from a tunable continuous-wave $(\mathrm{CW})$ laser source is injected though a fibrised polarisation controller and coupled to free space via a moulded aspheric lens mounted on a 3D translation stage. It is passed through a free space polariser and then coupled to the SOA using another lens and stage combination. The SOA output is collected using the same setup as for the ASE measurements. A polariser is not used at the output. The proportion of co-polarised ASE must be determined to calculate the NF, and is measured to be $85 \%$ (injecting along the dominant TE mode). The SOA is biased at $250 \mathrm{~mA}$. Initially, a full characterisation of the losses in the setup is performed taking into account the input and output mode coupling losses, reflections from coupling lens and mirror losses, as well as the loss associated with the input polariser. Total losses are approximately $8 \mathrm{~dB}$ for the input signal and $6-7 \mathrm{~dB}$ for the output signal. The NF is calculated using the following formula:

$$
N F=10 \log 10\left(\frac{2 \rho_{A S E}^{\prime \prime}}{G h v}\right)
$$

where $\rho_{A S E}^{\prime \prime}$ is the ASE power spectral density that is co-polarised with the signal, $\mathrm{G}$ is the optical gain, $\mathrm{h}$ is Planck's constant and $\mathrm{y}$ is the signal frequency.

Results and analysis: The NF profile as a function of the signal wave-length shows an inverse dependence on the gain owing to the correlation between the gain and nsp. The minimum NF of the NCSOA is centred on $1500 \mathrm{~nm}$ as seen in Fig. 3. Injecting a signal at the input of the device yields a minimum NF of $8.3 \mathrm{~dB}$. As expected, a higher NF of $11 \mathrm{~dB}$ is obtained for injection at the output facet of the device, as the gain clamped sections are now the first sections of the SOA. This difference 
of almost $3 \mathrm{~dB}$ can be clearly seen in Fig. 3, demonstrating the effectiveness of carrier density engineering on NF control.

Conclusions: A first generation novel noise controlled semiconductor optical amplifier has been demonstrated and characterised. The device exhibits a minimum noise figure of $8.3 \mathrm{~dB}$ at $1500 \mathrm{~nm}$ when injecting a signal into the optimal facet. This is $3 \mathrm{~dB}$ lower than the NF measured for a signal injected into the output facet. While our measured NF does not compare favourably to other reported values, it can be noted that this is a first generation device and superior performance can be expected with improvements in design, for example in terms of the etching of the grooves that comprise the lateral cavity. The concept of reduction of the noise figure by implementation of a lateral cavity along the output section of the SOA has been validated.

Acknowledgment: The authors thank Enterprise Ireland for supporting this research under grant number CFTD/06/IT/332. 


\section{References}

1 Mork, J., Nielsen, M.L., and Berg, T.W.: 'The dynamics of semiconductor optical amplifiers: modeling and applications', Opt. Photonics News, July 2003, pp. $42-48$

2 Senior, J.M.: 'Optical fibre communications: principles and practice' (Prentice Hall, 2008, 3rd edn), p. 783

3 Crottini, A., Salleras, F., Moreno, P., Dupertuis, M.-A., Deveaud, B., and Brenot, R.: 'Noise figure improvement in semiconductor optical amplifiers by holding beam at transparency scheme', IEEE Photonics

Technol. Lett., 2005, 17, (5), pp. $977-979$

4 Morito, K., Tanaka, S., Tomabechi, S., and Kuramata, A.: 'A broadband MQW semiconductor optical amplifier with high saturation output power and low noise figure', IEEE Photonics Technol. Lett., 2005, 17, (5), pp. 974 - 976

5 Friis, H.T.: 'Noise figures of radio receivers', Proc. IRE, 1944, 32, (7), pp. $419-422$

6 Durhuus, T., Mikkelsen, B., and Stubkjaer, K.E.: 'Detailed dynamic model for semiconductor optical amplifiers and their crosstalk and intermodular distortion', IEEE J. Lightwave Technol., 1992, 10, (8), pp. $1056-1065$

7 Landais, P., Patent no. GB0821602.0

8 Giuliani, G., and D'Alessandro, D.: 'Noise analysis of conventional and gain-clamped semiconductor optical amplifiers', IEEE J. Lightwave

Technol., 2000, 18, (9), pp. 1256- 1263

9 Bjorlin, E.S., and Bowers, J.E.: 'Noise figure of vertical-cavity semiconductor optical amplifiers', IEEE J. Quantum Electron., 2002, 38, (1), pp. 61 - 66 
Figures

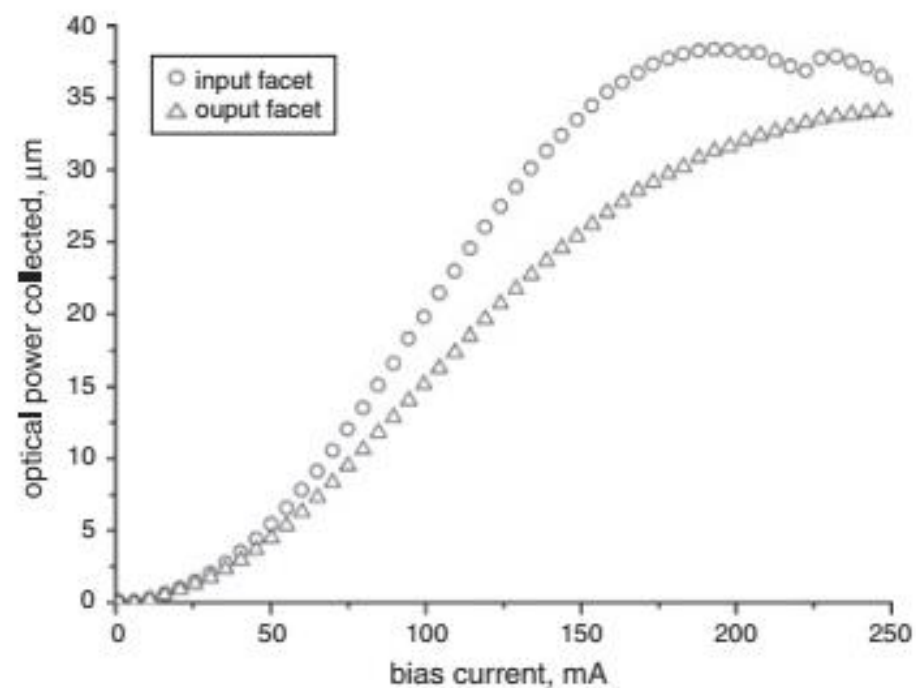

Fig. 1 ASE collected from both facets against bias current 

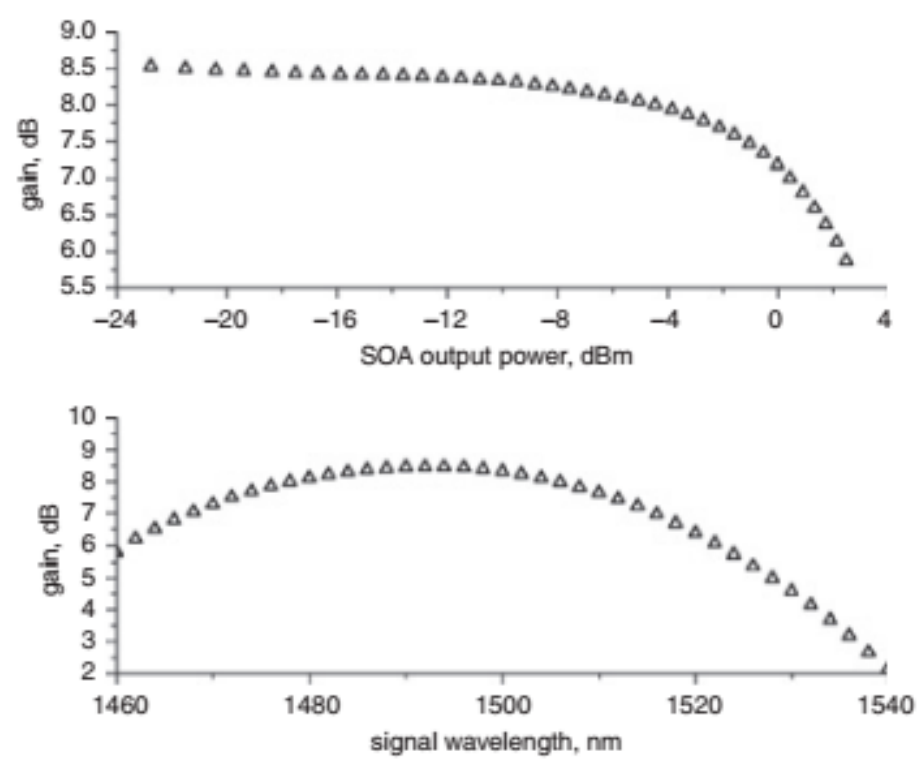

Fig. 2 Gain spectra of SOA against output power (top) at signal wavelength of $1510 \mathrm{~nm}$ and against input signal wavelength at input power of $210 \mathrm{dBm}$ (bottom) SOA biased at $250 \mathrm{~mA}$ 


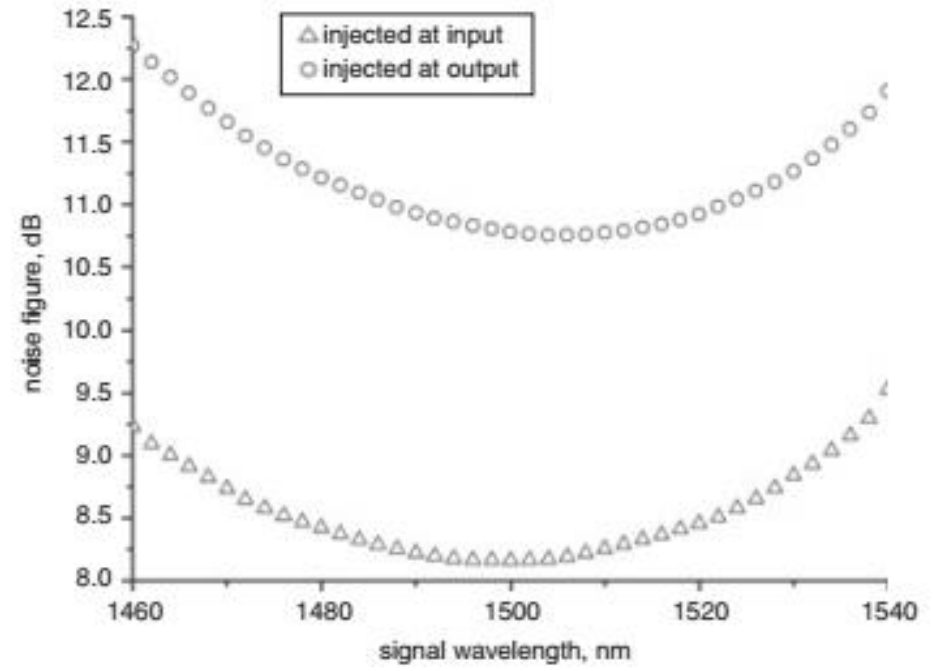

3 Noise figure of SOA against input signal wavelength.Input signal power $210 \mathrm{dBm}$ and SOA biased at $250 \mathrm{~mA}$ 